\title{
A compatible interaction of Alternaria brassicicola with Arabidopsis thaliana ecotype DiG: evidence for a specific transcriptional signature
}

\author{
Arup K Mukherjee ${ }^{1}$, Sophie Lev ${ }^{2}$, Shimon Gepstein ${ }^{2}$ and \\ Benjamin A Horwitz*2
}

Address: ${ }^{1}$ Division of Plant Biotechnology, Regional Plant Resource Centre, IRC Village, Bhubaneswar 751015, Orissa, India and ${ }^{2}$ Department of Biology, Israel Institute of Technology, Technion, Haifa 32000, Israel

Email: Arup K Mukherjee - titirtua@gmail.com; Sophie Lev - levsophie@gmail.com; Shimon Gepstein - gepstein@tx.technion.ac.il; Benjamin A Horwitz* - horwitz@tx.technion.ac.il

* Corresponding author

Published: 18 March 2009

BMC Plant Biology 2009, 9:31 doi:10.1 186/147I-2229-9-31
Received: 12 December 2008

Accepted: 18 March 2009

This article is available from: http://www.biomedcentral.com/I47I-2229/9/3I

(C) 2009 Mukherjee et al; licensee BioMed Central Ltd.

This is an Open Access article distributed under the terms of the Creative Commons Attribution License (http://creativecommons.org/licenses/by/2.0), which permits unrestricted use, distribution, and reproduction in any medium, provided the original work is properly cited.

\begin{abstract}
Background: The interaction of Arabidopsis with Alternaria brassicicola provides a model for disease caused by necrotrophs, but a drawback has been the lack of a compatible pathosystem. Infection of most ecotypes, including the widely-studied line Col-0, with this pathogen generally leads to a lesion that does not expand beyond the inoculated area. This study examines an ecotype, Dijon G (DiG), which is considered sensitive to A. brassicicola.
\end{abstract}

Results: We show that the interaction has the characteristics of a compatible one, with expanding rather than limited lesions. To ask whether DiG is merely more sensitive to the pathogen or, rather, interacts in distinct manner, we identified genes whose regulation differs between Col- 0 and $\mathrm{DiG}$ challenged with $\mathrm{A}$. brassicicola. Suppression subtractive hybridization was used to identify differentially expressed genes, and their expression was verified using semi-quantitative PCR. We also tested a set of known defense-related genes for differential regulation in the two plantpathogen interactions. Several known pathogenesis-related $(P R)$ genes are up-regulated in both interactions. $P R I$, and a monooxygenase gene identified in this study, $M O I$, are preferentially upregulated in the compatible interaction. In contrast, GLIPI, which encodes a secreted lipase, and $D I O X I$, a pathogen-response related dioxygenase, are preferentially up-regulated in the incompatible interaction.

Conclusion: The results show that DiG is not only more susceptible, but demonstrate that its interaction with $A$. brassicicola has a specific transcriptional signature.

\section{Background}

Alternaria brassicicola, the agent of black spot disease of crucifers, is able to infect Arabidopsis. Different ecotypes and genetic backgrounds show variation in susceptibility to this necrotrophic pathogen. Defenses against necro- trophs and biotrophs employ different mechanisms [1]. Programmed cell death and production of reactive oxygen species (ROS) are hallmarks of the hypersensitive response (HR) that is a means of plant defense against biotrophs $[2,3]$. Perception of the pathogen leads to rapid 
changes in expression of genes including receptor-like protein kinases, followed by cell death and HR-related defense [4-6]. Necrotrophs, in contrast, assimilate nutrients from dead host tissue, and actually benefit from ROS production and programmed cell death $[7,8]$. It was found, for example, that oxalic acid is apparently a virulence factor for Sclerotinia sclerotiorum because it signals for increased ROS production and programmed cell death in the plant [9]. Studies with Arabidopsis mutants in different hormone-dependent defense pathways showed that defense against necrotrophs primarily employs jasmonic acid and ethylene-dependent pathways $[10,11]$. Integration with SA-dependent pathways is also important, and there is cross-talk between the SA and JA pathways [1214]. An estimated $0.48 \%$ of the Arabidopsis transcriptome was induced two-fold or more in response to infection with the wide host-range necrotroph Botrytis cinerea, and the expression of these genes depends on ethylene, jasmonate and SA pathways [15]. Defense against necrotrophs thus does not necessarily follow the gene-for-gene pattern in which successful recognition implies triggering of the HR. Inoculation of Arabidopsis leaves with A. brassicicola generally leads to an incompatible interaction in which the lesion does not spread significantly beyond where the fungus was inoculated. In contrast, Brassica oleracea is a compatible host, and spreading necrotic lesions are formed. Extensive gene expression data are available for incompatible interactions between Arabidopsis and A. brassicicola [16-18]. Incompatible and compatible interactions with the bacterial pathogen Pseudomonas syringae were compared in a genome-wide study, and the conclusion was that the distinction is mainly a quantitative and kinetic one [19].

The molecular basis for the extent to which the plant can limit infection by necrotrophic fungi is of obviously of great interest, but the use of Arabidopsis genetics to investigate this question has been limited by the need to study compatible and incompatible pathosystems for the same pathogen species. Efforts to overcome this gap have begun for Colletotrichum-Arabidopsis and Leptosphaeria-Arabidopsis pathosystems [20,21]. Interactions with species of Botrytis have been studied at the cellular level, leading to a model in which resistance depends on the balance between cell death and survival [22]. A. brassicicola is an attractive system because of the considerable amount of work already done with this pathosystem. As for Leptosphaeria and Botrytis, there is a genome project for A. brassicicola [23], which is currently in the manual curation stage (Dothidiomycete group and the Joint Genome Institute, US Department of Energy, unpubl.). Arabidopsis mutants defective in biosynthesis of the antimicrobial compound camalexin are more susceptible to A. brassicicola [24-26]. The Dijon-G (DiG) ecotype is one of the most susceptible, and is a low-camalexin ecotype [27]. Additional factors are involved, because disease resistance did not directly correlate with camalexin levels in the 24 ecotypes studied [27]. Indeed, A. brassicicola infection of wild type and camalexin-deficient pad 3 mutant plants resulted in a generally similar transcriptional pattern [17]. A secreted lipase encoded by the Arabidopsis gene GLIP1 is important for resistance to A. brassicicola [28]. Analysis of the A. brassicicola-Brassica oleracea interaction led to the identification of a collection of A. brassicicola EST sequences characteristic of this compatible interaction [29]. The DiG-A. brassicicola pair, chosen to investigate the role of the $A$. brassicicola non-ribosomal peptide synthase gene NRPS6 as a virulence factor [30], has a compatible appearance. This led us to consider the question of whether there is merely a continuous range of susceptibility among ecotypes, or rather a fundamental difference between compatible and incompatible interactions. If one postulates that the DiG and Col-0 interactions with A. brassicicola differ merely in the extent of sensitivity of the plant to the pathogen, the transcriptional profiles should be very similar in the two interactions. The aim of this study was to ask whether the transcriptional profile of this particular $A$. brassicicola-Arabidopsis interaction differs from that of an incompatible interaction. Differential cDNA screening and a candidate gene approach led to the identification of specific markers for the two types of interaction. The hypothesis that the interactions are identical can thus be excluded.

\section{Results}

A total of five wild type ecotypes, Col-0, Col-6, DiG (Dijon G), Ler (Landsberg erecta), Ws (Wassilewskija) and three mutants (glip1-1, glip1-2 and acd1) were screened against A. brassicicola (Fig. 1a). These mutants were tested initially, because glip1 mutants were shown to be susceptible to A. brassicicola [28]. The mutant acd1 was chosen because we reasoned that, as LLS1 in maize and its ortholog ACD1 in Arabidopsis are required to limit the spread of cell death [31,32], loss of this gene might increase the spread of a necrotrophic pathogen. Inoculum amount and sampling times after inoculation were calibrated in preliminary experiments so that the different plant-fungal pairs could be compared under non-saturating conditions (data not shown). Lesion diameter and spore production were measured (Fig. 1b) to assess the disease progression. The Col-0 accession showed an incompatible interaction, in which the lesion did not progress beyond the boundaries of the inoculated region. Accession DiG was most susceptible, showing larger lesions than either of the glip1 mutants or Col-6, which are relatively susceptible as compared to Col-0 [28]. The lesion-mimic mutant acd1, was no more susceptible to the pathogen than was Col-0 (Fig. 1a,d). The lesions on DiG leaves continued to spread (Fig. 1c) and often show concentric rings (Fig. 1b), as seen in the interaction with the 


\section{a}

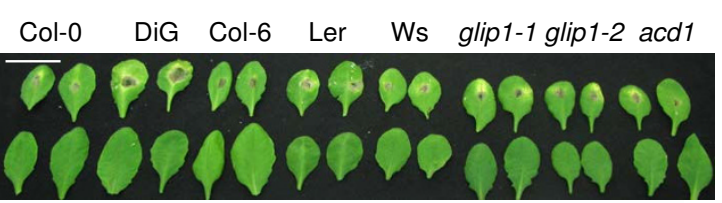

b

Col-0 DiG
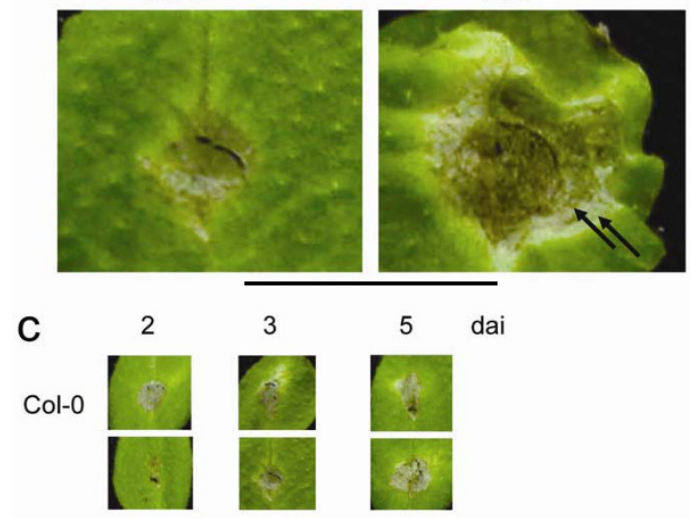

3
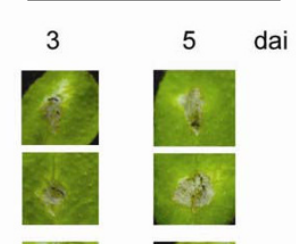

DiG

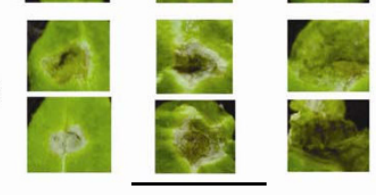

d
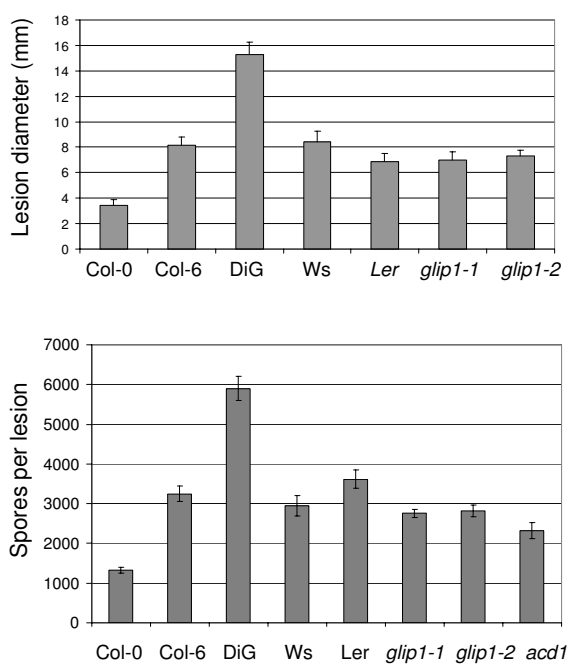

Figure I

Characterization of Arabidopsis-Alternaria brassicicola pairs. a) Symptoms in different ecotypes and genotypes, 3 days after inoculation of intact leaves. Top row, inoculated; bottom row, control. glip I-I and glip I-2 are two mutants at the glip I locus encoding a secreted lipase [28]; acdl is a lesion mimic mutant [32]. Scale bar indicates $2 \mathrm{~cm}$. b) Magnification of images of leaves from (a) showing the ring-like pattern in the progression of the lesion on a DiG leaf (arrows). The innermost dark, thin, arc (no arrow) is material from the inoculum. Scale bar indicates $1 \mathrm{~cm}$. c) Size of lesions on Col-0 and DiG leaves at different times post-inoculation. Representative infected leaves are shown, photographed at the indicated times after inoculation. Scale bar indicates $2 \mathrm{~cm}$. d) Quantitative analysis of lesion size and spore production. Top panel, lesion diameter was measured 5 days after inoculation. Error bars indicate standard errors of the mean for 7 replicate lesions (for Col-0, 9 and DiG, 10 replicates). Lower panel, lesions were excised 5 days after inoculation, the conidia suspended in water, and counted under the microscope in a hemocytometer chamber. Values are means of two independent experiments, consisting of 12 and 4-5 replicates, respectively; the error bars indicate the standard error of the mean of the combined data from the two experiments. 
compatible host Brassica oleracea but not in incompatible interactions with Arabidopsis. To test the possibility that the DiG-A. brassicicola interaction has a unique transcriptional signature, two approaches were followed: differential library screening and a candidate gene approach.

A suppression-subtractive hybridization ( $\mathrm{SSH}$ ) library was constructed to compare A. brassicicola-infected to mockinoculated leaves of ecotype DiG. In order to limit the set of ESTs selected, to the extent possible, to plant transcripts, cDNA from RNA isolated from a saprophytic culture of A. brassicicola was added to the driver population. In the SSH procedure, the driver competes with the differentially expressed transcripts. Furthermore, the library was constructed at $72 \mathrm{~h}$ post-infection, when most of the leaf was still green in both plant-fungal pairs (Fig. 1). Sequence was obtained for 116 clones (Table 1). The library was of high diversity: a chitinase clone, for example, was represented 5 times, and a glycosyl hydrolase three times in the sequences, but most transcripts were represented only once (Table 1 ). Almost all the sequences were identified in the Arabidopsis genome database. 14 of the genes in this set were already known to be up-regulated in response to $A$. brassicicola infection [17], and an additional transcript, PDIOX1, was also known to be upregulated in incompatible interactions [33]. Most of the genes, however, had not been identified previously in the interaction of A. brassicicola with Col-0 and its mutants. To determine whether these are all false positives or marginally up-regulated genes, or rather, represent a class of genes up-regulated in the compatible interaction, a set of primer pairs was designed based on sequences of nine clones representing transcripts that were not identified [17] in the interactions with Col-0 and its mutants, and which have annotated functions (Table 2). In an additional, candidate gene, approach, a set of known defenseresponse related genes (Table 2) was tested.

Semi-quantitative RT-PCR analysis of the abundance of the corresponding transcripts is shown in Fig. 2. An actin gene (ACT2), a ubiquitin-conjugating enzyme gene (UBC) and cap-binding protein 20 (CBP20) were used as "housekeeping" genes (Table 2). The false-positive library clones also serve as additional controls for overall efficiency of the RT PCR procedure (Fig. 2). The fold-induction by infection relative to Col-0 is shown in Fig. 3. The transcripts detected by three of the test primer pairs: PDIOX, RD21A and MO1F (Table 2) showed clearly differential expression in the compatible interaction. The transcripts corresponding to GS, MDH and PEX42 did not, although even a slight differential expression might have led to inclusion in the library. The transcript corresponding to primer pair PDIOX is more highly expressed in the incompatible interaction. The relatively low proportion of differential SSH clones in the test set (3 out of
9) may reflect the choice of test primer pairs, which included only genes that were not previously annotated as pathogen-response dependent (Table 1). Among the candidate genes tested, PR1, PR3, PR4 and PDF1.2 were strongly up-regulated in both Col-0 and DiG interactions. The transcript levels of these genes were very low in uninfected plants, with the exception of PR1 (Fig. 2), so that induction ratios could not be estimated. Transcripts of the other candidates (Table 1), including three ethyleneresponse related genes, were not detectable under these conditions and it can be inferred that they are not strongly induced. The infection-related lipase gene GLIP1 is expressed at a higher level (about 4-fold) in the incompatible than in the compatible interaction, while PR1 is expressed about 4-fold higher in the compatible than in the incompatible interaction.

\section{Discussion}

The A. brassicicola-DiG pathosystem has the features of a compatible interaction, producing expanding necrotic lesions. This suggests that there may be a fundamental difference between this interaction and an incompatible one, rather than merely a graded increase in sensitivity relative to Col-0. If this is so, the defense responses of the plant should differ between the compatible and incompatible interactions. As extensive transcriptional profiling has already been reported for incompatible A. brassicicola-Arabidopsis interaction (incompatible), an initial study of the A. brassicicola-DiG pathosystem was performed. The set of transcripts detected overlaps partially with those induced in resistant (Col-0) or relatively sensitive (pad3 in Col-0 background) interactions, but most of the SSH clones represent transcripts that had not been identified before as defense-related. Of a test set of 9 genes from the SSH library tested by RT-PCR, three were differentially expressed at 72 hai in the DiG-A. brassicicola interaction: the primer pair PDIOX (Fig. 2) corresponds to a gene which encodes an alpha-dioxygenase involved in protection against oxidative stress and cell death, and induced in response to salicylic acid and oxidative stress.

This gene, DIOX1, is preferentially induced in the incompatible interaction with $A$. brassicicola, in agreement with previous data for Arabidopsis-bacteria interactions [33]. Induction of the monooxygenase/aromatic-ring hydroxylase gene MO1 is specific to the compatible interaction. In Col-0 MO1 is not induced, and is present already in noninfected plants. This suggests that Col-0 may be "primed" in some way to initiate the defense response that is characteristic of infection with $A$. brassicicola. MO1 shares homology with monooxygenases that degrade SA. Overexpression of the SA-degrading enzyme NahG has been used to test the involvement of SA in defense responses, but the immediate product, catechol, may contribute to the phenotypes of NahG expressors [34]. The reaction cat- 
Table I: Randomly isolated SSH clones.

\begin{tabular}{|c|c|c|c|c|}
\hline Annotation & TAIR number & inc & primers & $x$ \\
\hline $60 S$ ribosomal protein $L I 3 A$ & AT3G24830.I & no & & \\
\hline SIR sulfite reductase & AT5G04590.I & yes & & 3 \\
\hline PSAL photosystem I subunit $L$ & $\overline{\text { AT4G } 12800.1}$ & no & & \\
\hline Cobalamin-independent methionine synthase & AT5GI7920.2 & no & MTH & \\
\hline glutamine synthase & $\overline{\text { ATIG66200.I }}$ & no & GS & 2 \\
\hline alpha dioxygenase I & AT3G0I420.I & no & PDIOX & \\
\hline NPQ4 non-photochemical quenching & $\overline{\text { ATIG44575.I }}$ & no & & \\
\hline protein phosphorylated amino acid binding & AT5G10450.2 & no & & 2 \\
\hline FF domain-containing protein $14-3-3$ stress related & AT3G19670.1 & no & & 2 \\
\hline sorbitol dehydrogenase & AT5G51970.2 & no & & 2 \\
\hline $26 \mathrm{~S}$ proteasome AAA-ATPase subunit & AT5G19990.1 & no & PATP & 2 \\
\hline \multicolumn{5}{|l|}{ no hit } \\
\hline APXI ascorbate peroxidase & ATIG07890.7 & yes & & \\
\hline acyl-CoA oxidase & AT5G65I10.2 & no & & \\
\hline chloroplastic drought-induced stress protein & ATIG76080.I & no & & \\
\hline chloroplast-encoded $23 \mathrm{~S}$ ribosomal & ATCG01180.1 & no & & 2 \\
\hline \multicolumn{5}{|l|}{ no hit } \\
\hline SHM3 serine hydroxymethyltransferase & AT4G32520.1 & no & & \\
\hline \multicolumn{5}{|l|}{ no hit } \\
\hline PDFI. 2 defensin & AT5G44420.I & yes & & \\
\hline Chitinase & AT2G43590.I & yes & & 6 \\
\hline \multicolumn{5}{|l|}{ no hit } \\
\hline lipid binding & ATIG04970.2 & no & & \\
\hline sugar transporter & $\overline{\text { ATIG77210.I }}$ & no & & \\
\hline \multicolumn{5}{|l|}{ no hit } \\
\hline LHCA4 Photosystem I light harvesting complex gene 4 & AT3G47470.1 & no & & \\
\hline ribosomal L6 & ATIG74050 & no & & \\
\hline dehalogenase hydrolase & AT2G32150.I & no & & \\
\hline expressed protein & $\overline{\text { ATIG07040.I }}$ & no & & \\
\hline protein kinase & $\overline{\text { AT2G23450.I }}$ & no & & \\
\hline NADPH cyt $\mathrm{P} 450$ reductase & AT4G24520.I & no & $\mathrm{P} 450$ & \\
\hline \multicolumn{5}{|l|}{ no hit } \\
\hline GTP binding & ATIGI7470.I & no & & \\
\hline GST & $\overline{\text { ATIG65820.I }}$ & no & & \\
\hline RuBisCo & AT5G38410.1 & no & & 2 \\
\hline cobalamin independent methionine synthase & AT3G03780.2 & no & & \\
\hline inorganic carbon transport, small stretch & $\overline{\text { AT4G32340.1 }}$ & no & & \\
\hline malate dehydrogenase & AT5G09660.2 & no & $\mathrm{MDH}$ & \\
\hline lipase class 3 & AT5G24210.1 & no & & \\
\hline \multicolumn{5}{|l|}{ no hits } \\
\hline glycosyl hydrolase family 17 & AT4G 16260.1 & yes & & 4 \\
\hline \multicolumn{5}{|l|}{ no hits } \\
\hline peroxidase 42 (PER42) & AT4G21960.1 & no & $\mathrm{PR} \times 42$ & \\
\hline speckle-type POZ protein-related & AT3G48360.1 & no & & \\
\hline UDP-glucose 4-epimerase & ATIGI2780.I & no & & \\
\hline cysteine proteinase $(\mathrm{RD} 2 \mid \mathrm{A}) /$ thio & ATIG47I28.I & no & RD2IA & \\
\hline chlorophyll A-B binding & AT3G61470.1 & no & & \\
\hline amino acid transporter family & AT3G56200.1 & no & & \\
\hline expressed protein & AT5G23040.2 & no & & \\
\hline glutamate:glyoxylate aminotransfer & ATIG 23310.2 & no & & \\
\hline expressed protein & AT5G02020.2 & no & & \\
\hline cysteine synthase, putative/O-ac & AT5G28030.I & no & & \\
\hline cytochrome b5 domain-containing & AT3G48890.I & yes & & \\
\hline protein kinase family protein & AT3G5I550.I & no & & \\
\hline chlorophyll A-B binding & ATIG6I520.2 & no & & \\
\hline sugar transport protein (STP4), & $\overline{\text { AT3G19930.1 }}$ & no & & \\
\hline expressed protein & AT5G54540.I & no & & \\
\hline glycine hydroxymethyltransferase & AT4G37930.1 & no & & \\
\hline similar to gamma-glutamylcysteine & $\overline{\text { AT4G23100.1 }}$ & yes & & \\
\hline
\end{tabular}


Table I: Randomly isolated SSH clones. (Continued)

\begin{tabular}{|c|c|c|c|c|}
\hline \multicolumn{5}{|l|}{ no hits } \\
\hline autophagy 7 (APG7) & AT5G45900.1 & no & & \\
\hline expressed protein & AT3G15450.3 & no & & \\
\hline expressed protein & AT4G19160.3 & no & & \\
\hline expressed protein & ATIG02475.I & no & & \\
\hline serine-rich protein-related & AT5G25280.2 & no & & \\
\hline GST & AT4G02520.1 & yes & & \\
\hline expressed protein & ATIG26II0.1 & no & & \\
\hline GSHI & AT4G23100.1 & yes & & \\
\hline meprin and TRAF homology domain & AT2G32870.1 & no & & \\
\hline PSBO2 & AT3G50820.1 & no & & \\
\hline actin-depolymerizing factor I & AT3G46010.1 & no & & \\
\hline spermidine synthase 2 & ATIG703I0.I & no & & \\
\hline cytochrome P450 (CYP83BI) & AT4G31500.1 & no & & 2 \\
\hline 2-oxoacid-dependent oxidase & AT3G49620.1 & no & & \\
\hline dehydrin (RABI8) & AT5G66400.1 & no & & \\
\hline expressed protein & ATIG64360.I & no & & \\
\hline auxin-responsive protein & AT3G07390.1 & no & & \\
\hline 3-oxoacyl-(acyl-carrier protein) & ATIG24360.I & no & $30 A$ & \\
\hline cysteine proteinase & AT5G60360.2 & no & & \\
\hline \multicolumn{5}{|l|}{ no hits } \\
\hline peroxisomal membrane $22 \mathrm{kDa}$ family & AT5G19750.I & no & & \\
\hline CBL-interacting protein kinase 6 & AT4G30960.1 & no & & \\
\hline coronatine-responsive protein & $\overline{\text { ATIGI9670.I }}$ & yes & & 2 \\
\hline hevein-like protein & AT3G04720.1 & yes & & \\
\hline monooxygenase (MOI), & AT4G15760.1 & no & MOI & \\
\hline KELP transcriptional coactivator pl5 & AT4G10920.1 & no & & \\
\hline peroxidase 42 (PER42) & AT4G21960.1 & no & & \\
\hline ubiquitin-conjugating enzyme I & ATIGI4400.2 & no & & \\
\hline mannitol transporter & AT4G36670 & yes & & \\
\hline aspartate aminotransferase 3 & AT5GII520.1 & no & & \\
\hline basic helix-loop-helix (bHLH) fami & AT5G46760.I & no & & \\
\hline expressed protein & $\overline{\text { AT4G25030.2 }}$ & yes & & \\
\hline no apical meristem (NAM) family & ATIG69490.I & no & & \\
\hline expressed protein & $\overline{\text { AT2GI5890.1 }}$ & no & & \\
\hline TMS membrane family protein & $\overline{\text { ATIGI6180.1 }}$ & no & & \\
\hline expressed protein & AT5G54730.1 & no & & \\
\hline $60 S$ ribosomal protein $L 23 \mathrm{~A}$ & AT2G39460.1 & no & & \\
\hline CBL-interacting protein kinase 6 & AT4G30960.1 & no & & \\
\hline Calmodulin & AT2G4I410.1 & yes & & \\
\hline RERIB & $\overline{\text { AT2G21600.1 }}$ & no & & \\
\hline glycine-rich RNA-binding protein & AT4GI3850.2 & no & & \\
\hline chlorophyll A-B binding & AT2G05070.1 & no & & \\
\hline cytochrome C & $\overline{\text { ATIG22840 }}$ & yes & & \\
\hline
\end{tabular}

The nucleotide sequences were used to search the TAIR database by BLASTN. A brief annotation and link to the TAIR database are given for each sequence. "No hits" indicates that no Arabidopsis gene was identified; these transcripts might represent fungal genes. The test primer pairs chosen are listed (names refer to Table 2), as well as the number of times ( $x$ ) that the same gene was identified in the set of cDNA clones sequenced (if more than once). Clones marked "yes" were previously reported [17] in an incompatible Arabidopsis-Alternaria interaction (inc).

alyzed by Mo1 is not known, but one possibility is that this enzyme produces SA-derived aromatic compounds that could have signaling roles. Another possibility is that Mo1 might be involved in the suppression of the SA pathway in Col-0. It is worthy of note that PR1 is more highly expressed in DiG, the opposite of what would be expected if Mo1 acts like NahG. GLIP1 shows the reverse pattern, and is induced less in the compatible interaction. This extracellular lipase-related protein contributes to resistance to A. brassicicola [28]. The decreased ability of DiG to upregulate GLIP1 in response to A. brassicicola may there- fore directly contribute to its sensitivity to the pathogen. DiG is a low producer of camalexin but this is probably not the only reason for its sensitivity, since other susceptible ecotypes produced up to several fold more camalexin than Col-0 [27]. Furthermore, the expression profiles of camalexin-lacking pad3 and wild type (Col-0) were similar and the data sets were indeed combined [17]. This contrasts with what was found here for DiG, suggesting that additional heritable traits are involved. Segregation of incompatible interaction - related traits in crosses between DiG and Col-0 may identify loci other than those 
Table 2: Test primer pairs used for semi quantitative RT-PCR amplification: names, TAIR database numbers, and predicted product sizes in bp are listed.

\begin{tabular}{|c|c|c|c|c|c|}
\hline name & function & TAIR ID & sense primer & antisense primer & $b p$ \\
\hline PATP & proteasome subunit & AT5GI9990 & GGCGTCCTGAGACAGCGATGGAG & CAGGCCTGAGAAGAGCTTGATCCAG & 938 \\
\hline GS & cytosolic glutamine synthetase & ATIG66200 & GAAGGATGTGAACTGGCCTCTTG & GTAAGGGTCCATGTTTGAAGCTG & 615 \\
\hline PDIOX & pathogen inducible dioxygenase & AT3G01420 & GTATGCGACGCCCTCAAGGATG & ССTTGAGACTCTCTGTAGTATTCACC & 936 \\
\hline MTH & homocysteine methyltransferase & AT5GI7920 & GCTGATCTCAGGTCATCCATCTG & GATTGAGCTTCTTCTGCTGAGCATC & 1170 \\
\hline $\mathrm{MDH}$ & malate dehydrogenase & AT5G09660 & GGAAAACTGCAGAGCTAAAGGTGG & CCAAGCTGATACACTTССТСTGC & 878 \\
\hline $\mathrm{PR} \times 42$ & peroxidase 42 & AT4G21960 & GACCACAACGAGAGTATCTCCGTC & CAAGCAGAGAACTCAACACACCAGAG & 544 \\
\hline RD2IA & cysteine proteinase (RD2IA) & ATIG47I 28 & GTGAGAGAAGGACTAGCCTACGGTAC & CACAAACCGGGTACTCGTGAG & 914 \\
\hline $30 A$ & 3-oxoacyl-(acyl-carrier protein) & ATIG24360 & GATGAAAACCGCTCTTGACAAATG & CATCAATGGTGAATGCCTGTCC & 511 \\
\hline MOI & aromatic-ring hydroxylase & AT4GI 5760 & GTTTGCTTGTCGTGCGGTGAGAG & CTGCACCGAAAGCCCGAGTAATC & 555 \\
\hline PRI & PRI pathogen related & AT2GI4610 & TCGTCTTTGTAGCTCTTGTAGGTG & TAGATTCTCGTAATCTCAGCTCT & 590 \\
\hline GLIPI & lipase, defense response & AT5G40990 & CGATTGTGCACCAGCCTCATTGGTT & CAGCGCTTTGAGATTATAGGGTCC & 429 \\
\hline PR2 & PR2 pathogen-related, cellulase & AT3G57260 & CGTTGTGGCTCTTTACAAACAACAAAAC & GAAATTAACTTCATACTTAGACTGTCGAT & 870 \\
\hline PR3 & PR3 defense response chitinase & AT3GI 2500 & CGGTGGTACTCCTCCTGGACCCACCGGC & CGGCGGCACGGTCGGCGTCTGAAGGCTG & 583 \\
\hline PR4 & PR4 hevein-like defense protein & AT3G04720 & GACAACAATGCGGTCGTCAAGG & AGCATGTTTCTGGAATCAGGCTGCC & 552 \\
\hline PR5 & PR5 thaumatin-like & ATIG75040 & ATGGCAAATATCTCCAGTATTCACA & ATGTCGGGGCAAGCCGCGTTGAGG & 484 \\
\hline PDFI.2 & defensin & AT5G44420 & GCTAAGTTTGCTTCCATCATCACCCTT & AACATGGGACGTAACAGATACACTTGT & 237 \\
\hline EIN2 & ethylene signal transduction & AT5G03280 & TGCAGCTCGCATAAGCGTTGTGACTGGTA & CGCTCTCTCCATTTAACCGAGTTAACAC & 379 \\
\hline EIN3 & ethylene signal transduction & AT3G20770 & GATGTTGATGAATTGGAGAGGAGGATG & ACGTCTCTGAGGAGGATCACAGTGT & 470 \\
\hline ERFI & ethylene response factor & AT3G23240 & CGGCTTCTCACCGGAATATTCTATCG & TCTCCGAAAGCGACTCTTGAACTCTCT & 415 \\
\hline ACT2 & actin 2 & AT3GI8780 & TCACCACAACAGCAGAGCGGG & GGACCTGCCTCATCATACTCGG & 257 \\
\hline UBC & ubiquitin conjugating & AT5G25760 & GGCATCAAGAGCGCGACTGTT & СTTTCTTAGGCATAGCGGCGAG & 217 \\
\hline CBP20 & Cap-binding protein 20 & AT5G44200 & TTGTGGCTTTTGTTTCGTCCTG & CGTGGGTTCTTCTCCGGTCTC & 409 \\
\hline
\end{tabular}

encoding camalexin biosynthesis genes. One candidate is GLIP1, and it would be of interest to construct a mutant lacking both GLIP1 and camalexin. Another candidate is the alpha dioxygenase DIOX1; the oxylipin signals produced by the alpha-DOX1 fatty acid dioxygenase encoded by this gene promote protection from ROS and cell death [33]. PR1, in contrast, is expressed at higher levels in DiG, despite the fact that this is a gene strongly induced by the SA pathway. This suggests that in DiG, the SA pathway might be induced upon infection with a necrotroph. Induction of the SA and JA pathways is coordinated, with induction of one pathway at the expense of the other $[13,14]$. Infection by a biotroph suppresses defense against the necrotroph A. brassicicola. Furthermore, the application of SA resulted in suppression of defense against the necrotroph, and high expression of the SAdependent defense gene PR1 [14]. The JA pathway is most important for defense against A. brassicicola [10]. Thus, induction of the SA pathway might be an important factor responsible for the development of a compatible interaction with DiG. It is possible that the extent of the trade-off between SA and JA-dependent pathways [14] has been modulated by selection in different plant ecotypes. It is striking that application of SA [14] closely mimicked the appearance of the compatible-type lesions that we observed in ecotype DiG (Fig. 1A-C). Likewise, we found strong induction of PR1 expression (Fig. 2). Expression of the JA-dependent defensin gene PDF1.2, however, was not strongly suppressed in DiG (Fig. 2), while application of SA strongly suppressed PDF1.2 expression over the entire 3-days post-inoculation period studied in [14]. Thus, suppression of JA-mediated defense may be only part of the explanation of the susceptible phenotype of DiG. An alternative explanation is that the reciprocal regulation of the SA and JA pathways might fail as a result of successful infection by the pathogen, making this an effect, rather than cause, of the difference between the ecotypes. Genes induced in infected as compared to control leaves (in both ecotypes) may have roles in defense responses, or be induced as a result of tissue damage and cell death. The set of ESTs identified by SSH includes known defense-regulated genes. These were not further tested for differential expression here, since they have been previously studied. These ESTs include: chitinase (At2g43590), glycosyl hydrolase family 17 (AT4G16260.1), sulfite reductase/ferredoxin (At5g04590.1), PDF2.1a (At5g44420.1), APX1 - ascorbate peroxidase (At1g07890.1), cytochrome b5 domaincontaining (AT3G48890.1), GST (AT4G02520.1), GSH1 (AT4G23100.1), coronatine responsive protein (chlorophyllase, methyl jasmonate induced, AT1G19670.1), hevein-like protein (HEL) (AT3G04720.1), mannitoltransporter (AT4G36670), calmodulin (AT2G41410.1), and cytochrome c (AT1G22840) (Table 1). Our identification of 15 known defense-regulated genes (about $15 \%$, taking into account redundancy in the set of sequenced clones) in the library (Table 1) shows that the SSH comparison was robust, despite only one third of the test set showing clearly differential expression between Col-0 and DiG (Fig. 2). We note that known A. brassicicola induced genes were excluded from the test set (Table 2). Although some sequences were redundant in the sample of more than 100 clones, the number of SSHderived ESTs apparently was not saturated with respect to 


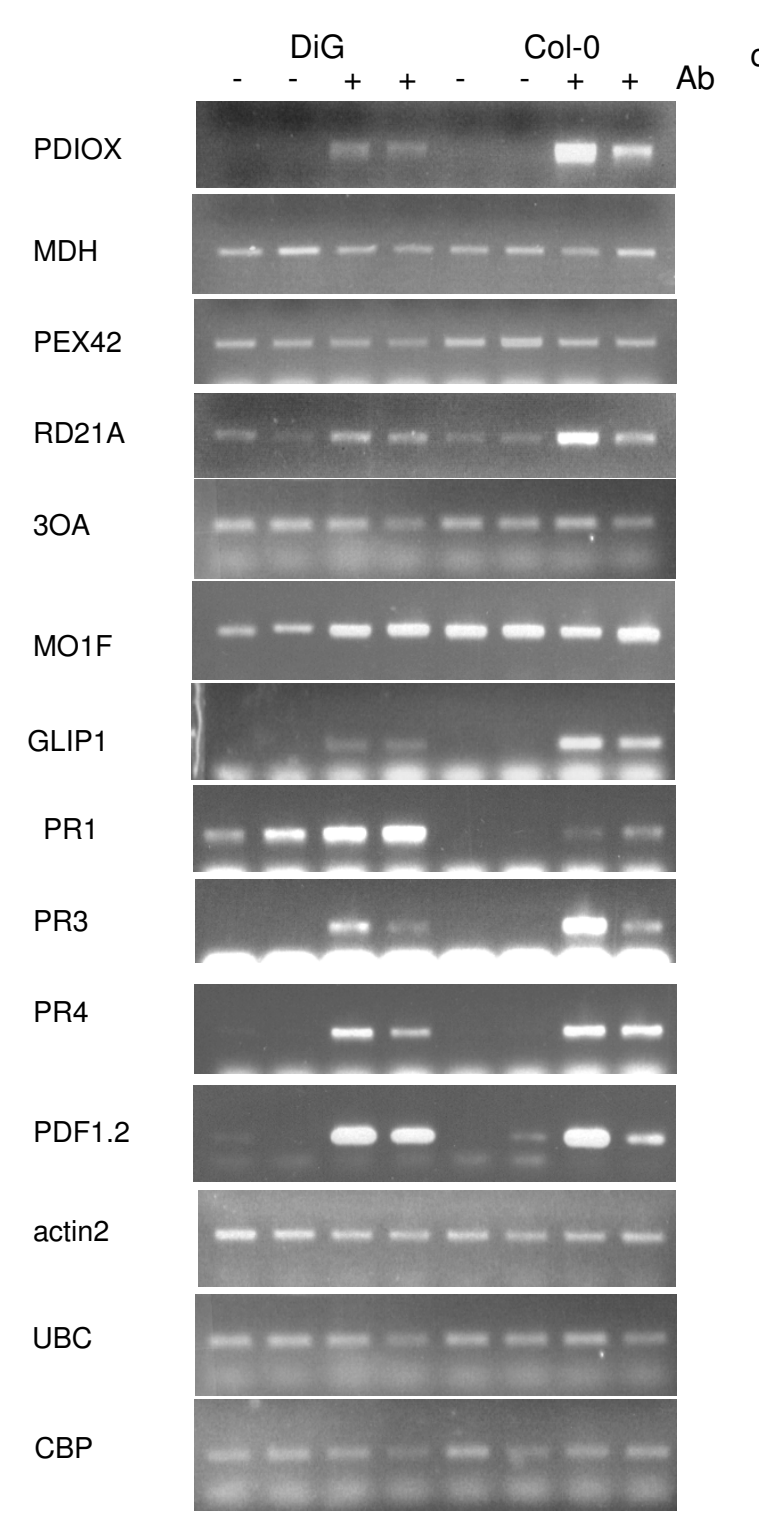

cycles

Figure 2

Semi-quantitative RT PCR analysis of transcript levels of selected genes. - and + indicate samples from control and inoculated intact plants, respectively. RNA was isolated by harvesting the entire leaf at $72 \mathrm{~h}$ after inoculation. Duplicate lanes indicate two independent experiments on different sets of plants; the number of amplification cycles is indicated at the right.

the number of clones sequenced. PDF1.2, for example, was strongly induced (Fig. 2) but not found among the sequenced clones.

\section{Conclusion}

The DiG-Alternaria brassicicola pathosystem shows all the characteristics of a compatible interaction between the necrotroph A. brassicicola and Arabidopsis. This initial study demonstrates that the transcriptional profile of the compatible interaction is not identical to that of the wellstudied incompatible interaction with Col-0. Induction of the monooxygenase gene MO1, and high expression and induction of PR1, are characteristic of the compatible interaction. GLIP1 and DIOX1, in contrast, are expressed more strongly in the incompatible interaction. These particular genes are not necessarily those whose expression levels define whether the plant is able to limit lesion spread or not, but are candidates for further study. The similarity between the compatible interaction and the result of exogenous application of SA provides a clue to the mechanism. Full-scale genome-wide studies are being done for the interaction of the hemibiotroph Magnaporthe oryzae with rice cultivars providing compatible or incompatible interactions $[35,36]$. This approach can now be fully developed for the Arabidopsis-Alternaria pathosystem defined in this study.

\section{Methods}

Plant material, growth conditions and RNA extraction

Seeds of the ecotypes Col-0, Col-6, WS and DiG were obtained from ABRC http://www.biosci.ohio-state.edu/ pcmb/Facilities/abrc/abrchome.htm. Mutants glip1-1, glip1-2 and acd1 were from the same collection, and homozygous lines were selected by screening progeny of selfed plants by PCR on genomic DNA samples using appropriate diagnostic primer pairs. Seeds were sown in "cookies" (approximately $4 \mathrm{~cm}$ diameter soil packaged in netting, purchased locally) held at $4{ }^{\circ} \mathrm{C}$ for two days to promote germination, and plants were grown in a temperature-controlled room at $23^{\circ} \mathrm{C}$ under continuous or $16 \mathrm{~h} /$ $8 \mathrm{~h}$ fluorescent lighting (cool-white tubes). Rosette leaves were inoculated with a conidial suspension of Alternaria brassicicola (MUCL20297, [10]). Inoculation was as described in [17], except that in preliminary experiments we found that a higher inoculum was needed to obtain reproducible disease development on both ecotypes, and so used $5 \mu \mathrm{l}$ of a $6 \times 10^{6}$ spore $/ \mathrm{ml}$ suspension $\left(3 \times 10^{4}\right.$ conidia per drop) throughout this study. To prepare spores for inoculation, fungal cultures were grown in a growth chamber on potato dextrose agar (Difco) plates under continuous white light at $25^{\circ} \mathrm{C}$ for 7 days, and spores suspended in water and counted in a haemocytometer. Plants were inoculated in a biosafety laminar flow chamber, placed in large sealed containers and incubated for up to 5 days at $23-24^{\circ} \mathrm{C}$ in a growth chamber. Conidia production was assayed after 5 days following inoculation by suspending the conidia from lesions excised from 10 infected leaves as described [17]. Leaf material was harvested, frozen, and kept at $-80^{\circ} \mathrm{C}$ until further use. RNA was extracted from control (mock inoculated) and inoculated leaves of Col-0 and DiG using Tri-Reagent (MBC or Fluka) according to the manufacturer's protocol, except that the starting material was leaf tissue ground in liquid 


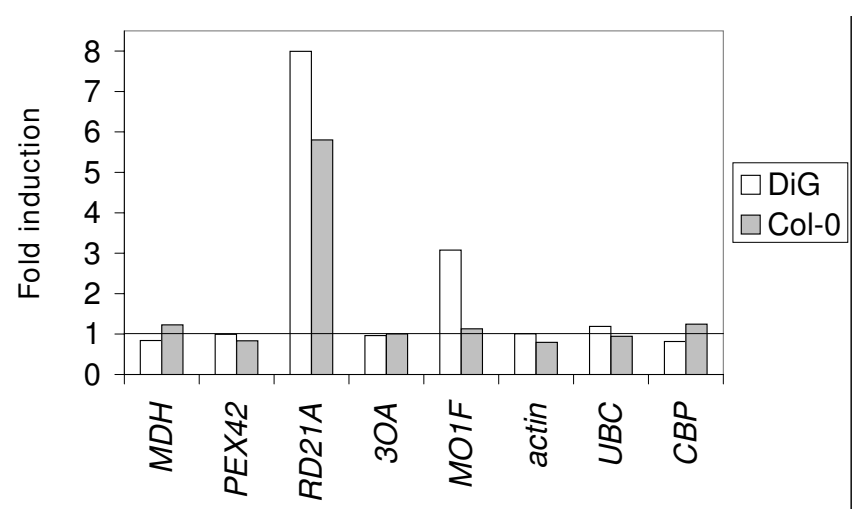

Figure 3

Expression of the monooxygenase gene $\mathrm{MOI}$ is preferentially up-regulated in the compatible interaction. Relative transcript levels were calculated from the intensity of the RT-PCR signals shown in Figure 2, as follows. Infected Col- 0 was chosen as the reference treatment. The band intensities of the three reference genes (ACT2, UBC and CBP20) then showed similar expression patterns as a function of experiment and replicate, over the entire data set, with no clear trend as a function of treatment. This indicates that the transcript levels of these genes varied with the amount of RNA and efficiency of the reactions, rather than with the treatment. All data for the reference genes were therefore combined, and the entire data set normalized to the combined reference values to obtain the signal plotted as "fold induction" (y-axis).

nitrogen. Each sample for RNA extraction consisted of about 20 leaves from at least 10 plants. (All the experiments were repeated at least three times with three replications) RNA concentrations were determined using a Nanodrop spectrophotometer and quality was checked by electrophoresis on denaturing agarose gels.

\section{Suppression-subtractive hybridization (SSH)}

4 week old DiG plants were inoculated with A. brassicicola with at least three replications and three biological repetitions. The infected leaves were collected after $6 \mathrm{~h}$ (Ast-1), $12 \mathrm{~h}$ (Ast-2), $24 \mathrm{~h}$ (Ast-3), $48 \mathrm{~h}$ (Ast-4) and $72 \mathrm{~h}$ (Ast-5) of inoculation. Total RNA was isolated from each stage of disease development along with control (Ast-0). The leaf samples were pooled for each stage from each replication and repetition of the experiment. For the SSH analysis the mRNA was enriched from the total RNA of Ast-0, Ast-3, Ast- 4 and Ast- 5 using the Qiagen Oligotex mRNA isolation Kit. SSH was performed using the Clontech PCRSelect cDNA subtraction kit following the manufacturer's protocol and the driver population consisted of mRNA from Ast-0 and A. brassicicola in the ratio of 3:1. A. brassicicola RNA was from a culture grown for 60 hours in shake culture on PDB. Amplification using primer pairs specific for the defense response gene PR 1 and an actin gene confirmed that the SSH procedure functioned as expected (data not shown). For this analysis, the following primers were used, spanning regions without RsaI sites: PR1 primer, sense direction, see Table 2; PR1 antisense, GATCACATCATTACTTCATTAGTATG. ACT2 primers: sense GCTGGATTCTGGTGATGGTG, antisense GATTCCAGCAGCTTCCATTC.

An enrichment of 64 fold for PR1 relative to ACT2 was estimated from these amplification data; it should be noted that this is likely to be the combined effect of suppression of ACT2 cDNA abundance and/or enrichment of $P R 1$, as expected from the design of the SSH subtraction method (see PCRselect manual, Promega, and literature cited therein). The fragments amplified in the second PCR reaction were cloned into pTZ57R/T (Fermentas), transformed into E. coli DH5a (HIT, Real Biotech), 130 positive clones were picked and the inserts amplified from the bacterial colonies using M-13 forward and reverse primers. Sequence was obtained for 116 clones (Macrogen, Seoul, Korea).

\section{Semiquantitative $R T$ PCR analysis}

cDNA was synthesized and assayed as follows. $2 \mu \mathrm{g}$ of total RNA from rosette leaves of DiG or Col-0 plants inoculated as described above were treated with 2 units of RQ1 RNAse-free DNAse (Promega) in a volume of $10 \mu \mathrm{l}$. After addition of stop solution and incubation for $10 \mathrm{~min}$ at $65^{\circ} \mathrm{C}$, the sample was denatured in the presence of $0.5 \mu \mathrm{g}$ of oligo dT primer, cooled, 200 units of MMLV reverse transcriptase (Promega), 24 units of PRI RNAse inhibitor (PRI, TaKaRa) and dNTPs to a final concentration of 0.5 $\mathrm{mM}$ each were added, and the reaction volume adjusted to a total of $25 \mu \mathrm{l}$ in $1 \times \mathrm{MMLV}$ reaction buffer. cDNA synthesis was for $1 \mathrm{~h}$ at $42^{\circ} \mathrm{C}$. All reactions were carried out in a thermal cycler (Biometra). A set of three "housekeeping" gene primer pairs (Sigma) was used to calibrate template amount (Table 2). The cDNA samples were diluted such that similar signal intensity was obtained upon amplification with the Actin 2 (ACT2) primer pair (Table 2 ), and the number of cycles was calibrated for each primer pair in order for the amplification level to remain below saturation.

\section{Abbreviations}

dai: days after inoculation; hai: hours after inoculation; ROS: reactive oxygen species; HR: hypersensitive response; SA: salicylic acid; JA: jasmonic acid

\section{Authors' contributions}

AKM conceived of the study together with the other authors, and carried out the major part of the experiments. SL brought the compatible interaction phenotype of DiG to the attention of AKM and BAH, and participated in library construction and data analysis. SG participated in coordination and analysis of the results. BAH drafted 
the manuscript and carried out some of the gene expression experiments. All authors participated in writing the final manuscript. All authors read and approved the final manuscript.

\section{Author information}

AKM is a plant molecular geneticist interested in plant pathology and stress physiology. SG leads a group studying gene expression and control of leaf senescence and stress responses in Arabidopsis and other plants. BAH lab focuses on signal transduction genes of filamentous fungi including Dothidiomycete pathogens of plants. SL, former member of BAH lab and currently a postdoc at UC Berkeley, studies plant-pathogen interactions by molecular genetic approaches.

\section{Acknowledgements}

The authors are grateful to the Department of Biotechnology, Government of India for providing the DBT Overseas Associateship to A.K.M. This work was supported by the Technion Vice-President for Research (VPR) Fund, which provides funding for pilot studies.

\section{References}

I. Glazebrook J: Contrasting mechanisms of defense against biotrophic and necrotrophic pathogens. Annu Rev Phytopathol 2005, 43:205-227.

2. Nimchuk Z, Eulgem T, Holt BF 3rd, Dangl JL: Recognition and response in the plant immune system. Annu Rev Genet 2003, 37:579-609.

3. Jones DA, Thomas CM, Hammond-Kosack KE, Balint-Kurti PJ, Jones JD: Isolation of the tomato Cf-9 gene for resistance to Cladosporium fulvum by transposon tagging. Science 1994, 266(5 I 86):789-793.

4. Acharya BR, Raina S, Maqbool SB, Jagadeeswaran G, Mosher SL, Appel HM, Schultz JC, Klessig DF, Raina R: Overexpression of CRK 13, an Arabidopsis cysteine-rich receptor-like kinase, results in enhanced resistance to Pseudomonas syringae. Plant J 2007, 50(3):488-499.

5. Rowland O, Ludwig AA, Merrick CJ, Baillieul F, Tracy FE, Durrant WE, Fritz-Laylin L, Nekrasov V, Sjolander K, Yoshioka H, et al.: Functional analysis of Avr9/Cf-9 rapidly elicited genes identifies a protein kinase, ACIKI, that is essential for full Cf-9-dependent disease resistance in tomato. Plant Cell 2005, I 7(I):295-3I 0.

6. Kim ST, Kim SG, Hwang DH, Kang SY, Kim HJ, Lee BH, Lee JJ, Kang $\mathrm{KY}$ : Proteomic analysis of pathogen-responsive proteins from rice leaves induced by rice blast fungus, Magnaporthe grisea. Proteomics 2004, 4(I I):3569-3578.

7. Govrin EM, Levine $A$ : The hypersensitive response facilitates plant infection by the necrotrophic pathogen Botrytis cinerea. Curr Biol 2000, I O(13):75I-757.

8. Mayer AM, Staples RC, Gil-ad NL: Mechanisms of survival of necrotrophic fungal plant pathogens in hosts expressing the hypersensitive response. Phytochemistry 200I, 58(I):33-4I.

9. Kim KS, Min JY, Dickman MB: Oxalic acid is an elicitor of plant programmed cell death during Sclerotinia sclerotiorum disease development. Mol Plant Microbe Interact 2008, 2 I (5):605-6I 2.

10. Thomma BPHJ, Eggermont IAMA, Penninckx B, Mauch-Mani B, Vogelsang R, Cammue BPA, Broekaert WF: Separate jasmonatedependent and salicylate-dependent defense-response pathways in Arabidopsis are essential for resistance to distinct microbial pathogens. Proc Natl Acad Sci USA 1998, 95(25): $|5| 07-15|| \mid$.

II. Coego A, Ramirez V, Gil MJ, Flors V, Mauch-Mani B, Vera P: An Arabidopsis homeodomain transcription factor, OVEREXPRESSOR OF CATIONIC PEROXIDASE 3, mediates resistance to infection by necrotrophic pathogens. Plant Cell 2005, I7(7):2123-2137.
12. Ferrari S, Plotnikova JM, De Lorenzo G, Ausubel FM: Arabidopsis local resistance to Botrytis cinerea involves salicylic acid and camalexin and requires EDS4 and PAD2, but not SID2, EDS5 or PAD4. Plant J 2003, 35(2): 193-205.

13. Spoel SH, Koornneef A, Claessens SM, Korzelius JP, Van Pelt JA, Mueller MJ, Buchala AJ, Metraux JP, Brown R, Kazan K, et al.: NPR I modulates cross-talk between salicylate- and jasmonatedependent defense pathways through a novel function in the cytosol. Plant Cell 2003, I5(3):760-770.

14. Spoel SH, Johnson JS, Dong X: Regulation of tradeoffs between plant defenses against pathogens with different lifestyles. Proc Natl Acad Sci USA 2007, I 04(47): I 8842- 18847.

15. AbuQamar S, Chen X, Dhawan R, Bluhm B, Salmeron J, Lam S, Dietrich RA, Mengiste $T$ : Expression profiling and mutant analysis reveals complex regulatory networks involved in Arabidopsis response to Botrytis infection. Plant J 2006, 48(I):28-44.

16. Schenk P, Kazan K, Wilson I, Anderson J, Richmond T, Somerville S, Manners J: Coordinated plant defense responses in Arabidopsis revealed by microarray analysis. Proc Natl Acad Sci USA 2000, 97:11655-11660.

17. van Wees SC, Chang HS, Zhu T, Glazebrook J: Characterization of the early response of Arabidopsis to Alternaria brassicicola infection using expression profiling. Plant Physiol 2003, I32(2):606-617.

18. Narusaka Y, Narusaka M, Seki M, Ishida J, Nakashima M, Kamiya A, Enju A, Sakurai T, Satoh M, Kobayashi M, et al.: The cDNA microarray analysis using an Arabidopsis pad3 mutant reveals the expression profiles and classification of genes induced by Alternaria brassicicola attack. Plant Cell Physiol 2003, 44(4):377-387.

19. Tao Y, Xie Z, Chen W, Glazebrook J, Chang HS, Han B, Zhu T, Zou G, Katagiri F: Quantitative nature of Arabidopsis responses during compatible and incompatible interactions with the bacterial pathogen Pseudomonas syringae. Plant Cell 2003, I5(2):317-330.

20. O'Connell R, Herbert C, Sreenivasaprasad S, Khatib M, EsquerreTugaye MT, Dumas B: A novel Arabidopsis-Colletotrichum pathosystem for the molecular dissection of plant-fungal interactions. Mol Plant Microbe Interact 2004, I 7(3):272-282.

21. Bohman S, Staal J, Thomma BP, Wang M, Dixelius C: Characterisation of an Arabidopsis-Leptosphaeria maculans pathosystem: resistance partially requires camalexin biosynthesis and is independent of salicylic acid, ethylene and jasmonic acid signalling. Plant $J$ 2004, 37(I):9-20.

22. van Baarlen $P$, Woltering $E$, Staats $M$, van Kan J: Histochemical and genetic analysis of host and non-host interactions of Arabidopsis with three Botrytis species: an important role for cell death. Molec Plant Pathol 2007, 8(I):4I-54.

23. Cramer RA, Lawrence CB: Identification of Alternaria brassicicola genes expressed in planta during pathogenesis of Arabidopsis thaliana. Fungal Genet Biol 2004, 4 I (2): I I5-I28.

24. Thomma BP, Nelissen I, Eggermont K, Broekaert WF: Deficiency in phytoalexin production causes enhanced susceptibility of Arabidopsis thaliana to the fungus Alternaria brassicicola. Plant J 1999, 19(2):163-171.

25. Nafisi M, Goregaoker S, Botanga CJ, Glawischnig E, Olsen CE, Halkier BA, Glazebrook J: Arabidopsis cytochrome P450 monooxygenase 7 IAI3 Catalyzes the conversion of indole-3-acetaldoxime in camalexin synthesis. Plant Cell 2007, I 9(6):2039-2052

26. Schuhegger R, Nafisi M, Mansourova M, Petersen BL, Olsen CE, Svatos A, Halkier BA, Glawischnig E: CYP7 IB I 5 (PAD3) catalyzes the final step in camalexin biosynthesis. Plant Physiol 2006, I4I(4): I248-I 254.

27. Kagan IA, Hammerschmidt R: Arabidopsis ecotype variability in camalexin production and reaction to infection by Alternaria brassicicola. J Chem Ecol 2002, 28(I I):2 I II-2I 40.

28. Oh IS, Park AR, Bae MS, Kwon SJ, Kim YS, Lee JE, Kang NY, Lee S, Cheong H, Park OK: Secretome analysis reveals an Arabidopsis lipase involved in defense against Alternaria brassicicola. Plant Cell 2005, I7( I 0):2832-2847.

29. Cramer RA, Mauricio la Rota C, Cho Y, Thon M, Craven KD, Knudson DL, Mitchell TK, Lawrence CB: Bioinformatic analysis of expressed sequence tags derived from a compatible Alternaria brassicicola-Brassica oleracea interaction. Molec Plant Pathol 2006, 7(2): II3-124. 
30. Oide S, Moeder W, Krasnoff S, Gibson D, Haas H, Yoshioka K, Turgeon BG: NPS6, encoding a nonribosomal peptide synthetase involved in siderophore-mediated iron metabolism, is a conserved virulence determinant of plant pathogenic ascomycetes. Plant Cell 2006, I 8( (10):2836-2853.

31. Gray J, Close PS, Briggs SP, Johal GS: A novel suppressor of cell death in plants encoded by the Lls I gene of maize. Cell 1997, 89(1):25-31.

32. Yang M, Wardzala E, Johal GS, Gray J: The wound-inducible LIs I gene from maize is an orthologue of the Arabidopsis Acd I gene, and the LLSI protein is present in non-photosynthetic tissues. Plant Mol Biol 2004, 54(2): I75-I9|.

33. De Leon IP, Sanz A, Hamberg M, Castresana C: Involvement of the Arabidopsis alpha-DOXI fatty acid dioxygenase in protection against oxidative stress and cell death. Plant J 2002, 29(I):6I-62.

34. van Wees SC, Glazebrook J: Loss of non-host resistance of Arabidopsis NahG to Pseudomonas syringae pv. phaseolicola is due to degradation products of salicylic acid. Plant J 2003, 33(4):733-742.

35. Lu G, Jantasuriyarat C, Zhou B, Wang GL: Isolation and characterization of novel defense response genes involved in compatible and incompatible interactions between rice and Magnaporthe grisea. Theor Appl Genet 2004, 108(3):525-534.

36. Ribot C, Hirsch J, Balzergue $S$, Tharreau D, Notteghem JL, Lebrun $\mathrm{MH}$, Morel JB: Susceptibility of rice to the blast fungus, Magnaporthe grisea. J Plant Physiol 2008, 165(I): I |4-I24.

Publish with Bio Med Central and every scientist can read your work free of charge

"BioMed Central will be the most significant development for disseminating the results of biomedical research in our lifetime. "

Sir Paul Nurse, Cancer Research UK

Your research papers will be:

- available free of charge to the entire biomedical community

- peer reviewed and published immediately upon acceptance

- cited in PubMed and archived on PubMed Central

- yours - you keep the copyright

Submit your manuscript here:

http://www.biomedcentral.com/info/publishing_adv.asp
BiolMedcentral 